\title{
Vulnerabilidad y cronificación de la pobreza: estudio de caso de la exclusión social en Olot (Girona)
}

\author{
Vulnerability and chronification of poverty: \\ A case study of social exclusion in Olot (Girona)
}

EDUARD CARRERA

Universitat de Girona

QUIM BRUGUÉ

Universitat de Girona

XAVIER CASADEMONT

Universitat de Girona

\section{Cómo citar/Citation}

Carrera, E., Brugué, Q. y Casademont, X. (2021). Vulnerabilidad y cronificación de la pobreza: estudio de caso de la exclusión social en Olot (Girona). Revista Española de Ciencia Política, 57, 219-242. Doi: https://doi.org/10.21308/recp.57.08

\section{Resumen}

Nos encontramos en un contexto donde se han extendido, intensificado y transformado las situaciones de pobreza y exclusión social. Este contexto muestra una realidad más compleja y diversa que la habitual y que, en consecuencia, desafia las formas tradicionales de construir e implementar las políticas sociales. Aunque el debate es amplio, este artículo se centra en la aparición de dos fenómenos centrales para definir la actual realidad social: la cronificación de la probreza y el incremento de vulnerabilidad social. En primer lugar, situaremos estos dos fenómenos en un marco conceptual sobre la exclusión social; a continuación, examinaremos su manifestación empírica en un caso concreto:la ciudad de Olot, en la provincia de Girona. Esto servirá para valorar hasta qué punto estos nuevos conceptos (cronificación y vulnerabilidad) son útiles para presentar la realidad social de una comunidad concreta y, simultáneamente, observar cómo los debates conceptuales se traducen en desafíos prácticos.

Palabras clave: cronificación, pobreza, vulnerabilidad, exclusión social, políticas sociales, servicios sociales, política local.

\section{Abstract}

We are in a context where situations of poverty and social exclusion have spread, intensified and transformed. This context shows a more complex and diverse reality than the usual one; which, 
in turn, challenges the traditional ways of defining and implementing social policies. Although the debate is extensive, this article focuses on the emergence of two central phenomena to define the current social reality: the chronification of poverty and an increase in social vulnerability. First, these two phenomena will be placed in a conceptual framework on social exclusion; next, their empirical manifestation will be examined in a specific case: the city of Olot in the province of Girona). This will serve to assess the extent to which these new concepts (chronification and vulnerability) are useful to present the social reality of a specific community and, simultaneously, to observe how conceptual debates translate into practical challenges.

Keywords: chronification, poverty, vulnerability, social exclusion, social policies, social services, local policy.

\section{CONTEXTO, OBJETIVOS Y ESTRUCTURA}

Los debates sobre la exclusión social han proliferado en un contexto socioeconómico donde no solo se han extendido e intensificado algunas situaciones de pobreza, sino que también se han transformado. Es decir, no solo disponemos de datos que acreditan un incremento de la exclusión social, sino que, además, observamos situaciones, perfiles y trayectorias de exclusión inéditas hasta hace pocos años (Eurostat, 2019). Conceptos como vulnerabilidad o cronificación sintetizan algunas de las novedades: por un lado, personas que trabajan, pero en unas condiciones tan precarias que los sitúan en una zona muy cercana al abismo de la exclusión (vulnerabilidad) y, por otro lado, personas que parecen ya condenadas, sin posibilidades de salida, a esta situación de exclusión (cronificación).

En este artículo pretendemos dotarnos de referencias empíricas que nos permitan contrastar las aportaciones teóricas sobre estas nuevas manifestaciones de la exclusión social. Para ello nos basaremos en un estudio de caso sobre la exclusión social, así como de las políticas desplegadas para hacerle frente, realizado en una comarca del nordeste de España. Así pues, los objetivos de este artículo pueden resumirse en dos:

- Por un lado, utilizaremos la extensa literatura sobre nuevas formas de exclusión social para proponer un marco conceptual sobre la cronificación de la pobreza y la creciente vulnerabilidad de sectores cada vez más amplios de la población. Se trata de conceptos que han aparecido con fuerza y que sirven para acercarse a una realidad social que se ha transformado intensamente durante las últimas décadas. Mientras la pobreza se percibía tradicionalmente como una situación que, con esfuerzo y trabajo, podía superarse, hoy hablamos de los riesgos de caer por la pendiente de la exclusión y de las barreras, a veces insalvables, para superarlos. Vulnerabilidad y cronicidad son dos términos que usamos para resumir estos profundos cambios.

- Por otro lado, utilizaremos el estudio de caso de la ciudad de Olot (La Garrotxa, Girona) para contrastar el marco conceptual con una realidad empírica en un 
territorio concreto y, de este modo, profundizar en una realidad socioeconómica que hoy se expresa de maneras muy diversas y fragmentadas. En otros términos, el estudio de caso nos permitiría acercarnos a una realidad social específica, usando los conceptos de la cronificación y la vulnerabilidad. Podremos, de esta manera, valorar tanto la aplicabilidad como la relevancia de estos conceptos a un estudio empírico.

Así pues, nuestros objetivos son modestos. No pretendemos responder al porqué de estas nuevas dinámicas sociales ni, por lo tanto, disponemos de hipótesis sobre sus orígenes y sus consecuencias. Tan solo pretendemos presentar los rasgos que definen la situación social de un territorio concreto, usando nuevas herramientas conceptuales. Además, trascendiendo el estudio empírico, pretendemos comprobar la fortaleza de un nuevo marco conceptual que nos ayude a definir y comprender aquello que, a nuestro entender, es una realidad social emergente.

Para alcanzar los objetivos propuestos, hemos articulado el resto del texto en tres apartados. En primer lugar, presentamos un marco conceptual donde empezamos contextualizando el tránsito desde la pobreza hacia la exclusión para, a continuación, definir las tres dimensiones de la exclusión como situación, como riesgo y como proceso. Las dos primeras dimensiones nos permiten introducir y definir respectivamente los conceptos de cronicidad y vulnerabilidad. En segundo lugar, presentaremos el caso de estudio donde, usando los datos provenientes de una encuesta y de un amplio trabajo cualitativo, dotamos de contenido empírico a los conceptos de cronicidad y vulnerabilidad, al tiempo que los usamos para entender la realidad social de un territorio concreto. Finalmente, en las conclusiones valoramos hasta qué punto los conceptos nos han sido útiles para entender la realidad y, simultáneamente, identificamos algunos de los retos de futuro que se derivan de esta lectura conceptual y empírica.

\section{DE LA POBREZA A LA VULNERABILIDAD Y CRONIFICACIÓN}

De entrada, como apunta Bregman (2017), vivimos una época donde la riqueza y la superabundancia han generado, paradójicamente, un mundo inhóspito. Tras un período de crecimiento económico sin precedentes, observamos también cómo la pobreza y las desigualdades se mantienen o incluso se intensifican. Son muchos los estudios y los informes que nos ofrecen datos sobre esta realidad (Fernández Maíllo, 2019; Belzunegui y Valls, 2019). Se calcula que en España, con cifras de 2014, un $22 \%$ de la población vivía bajo el umbral de pobreza y 5 millones de personas en situaciones de exclusión social extrema, mientras que en la UE ya se contabilizaban 120 millones de personas pobres (Malgesini, 2014). Se trata de un problema que se agrava por los déficits redistributivos y por el consecuente incremento de la desigualdad social (Piketty, 2008).

La lucha contra la pobreza aparece en la agenda de prioridades de la Comisión Europea a través del programa 2020, aunque de momento su eficacia ha sido escasa. Lo que sí ha proliferado es un debate — académico y profesional— sobre el significado del término 
pobreza y su evolución hacia el concepto de exclusión social (Madanipour et al., 2015; Oosterlynck et al., 2019; Béland, 2007; Daly, 2006). Sin ser un término de nuevo cuño, el uso que hoy le damos al término exclusión pretende ir más allá de la pobreza, definiéndose como la imposibilidad o la dificultad de acceder a unos mínimos de protección social, desarrollo personal e inserción comunitaria (Sen, 1984: 342). Podríamos destacar dos aspectos que permiten diferenciar ambos conceptos con un amplio nivel de acuerdo.

El concepto de pobreza tiende a centrarse en aspectos económicos (ingresos insuficientes), mientras la exclusión incorpora déficits en ámbitos tan variados como la educación, la salud, la vivienda, la situación administrativa o las relaciones con entornos familiares y comunitarios. De este modo, frente a la unidimensionalidad de la pobreza, la exclusión se presenta como un fenómeno complejo y multidimensional (Percy-Smith, 2000; Pierson, 2002) que presentan los problemas sociales y que complementa el análisis de las desigualdades y la falta de ingresos con otra perspectiva (Laparra y Pérez, 2008). Por otra parte, tendemos a entender la pobreza como una situación estática en la que se encuentran determinadas personas, mientras que el concepto de exclusión incorpora una visión dinámica; es decir,una perspectiva que, más allá de las situaciones individuales, incorpora tanto los procesos como los riesgos de caer en situaciones de pobreza (Townsend, 2010; Tezanos, 1999), y que permite incluir tres aspectos clave: el origen estructural, el carácter multidimensional y su naturaleza dinámica (Laparra y Pérez, 2008).

Así pues, en un contexto de creciente complejidad y heterogeneidad, la exclusión no se refiere únicamente a las tradicionales desigualdades unidimensionales y verticales de la sociedad industrial, sino que incorpora múltiples ejes que explican situaciones e itinerarios de exclusión diversos (Ginsburg, 1992; Hulme y Shepherd, 2003). Solíamos interpretar la desigualdad desde la lógica arriba-abajo; es decir, diferenciando las clases altas de las clases bajas en términos tanto de recursos materiales como de referentes político-simbólicos. Estas diferencias siguen siendo importantes, aunque las políticas clásicas del Estado de bienestar las han amortiguado a través de un conjunto de prestaciones que garantizan unos mínimos de seguridad material (Adelantado, 2000; Moreno, 2000). Sin embargo, esta situación de seguridad ha desaparecido con la llegada del nuevo milenio (Piketty, 2020). Las transformaciones que explican esta nueva situación son múltiples, pero destacamos dos aspectos de carácter estructural:

- Por un lado, la consolidación de las políticas redistributivas del Estado de bienestar provocaron la paulatina reducción de las tradicionales segmentaciones verticales en términos de clase social. La mayoría de la población dejó de identificarse según estas categorías clásicas y emergió una borrosa nueva clase media que, siguiendo los estudios de Inglehart (1991), una vez superadas sus necesidades materiales básicas, concentraba su atención en demandas y expectativas "posmaterialistas». Se trata de una población que ha alcanzado ciertos niveles de seguridad y que proyecta sus expectativas hacia unos servicios más personalizados y de mayor calidad; ya no se conforma con prestaciones universales, sino que reclama respuestas flexibles y adaptadas a sus situaciones personales. Pero también se trata 
de una población que ha ido reconociendo de forma creciente su fragilidad, su vulnerabilidad. Afirmaba Beck (1992) que las viejas estabilidades habían dejado paso a una democratización de la exclusión social; una «sociedad del riesgo» donde emerge un colectivo cada vez más amplio e inédito para las políticas sociales de personas que, sin ser usuarias de servicios sociales en el presente, son potenciales usuarias en el futuro. Un colectivo desconcertante, tanto a la hora de valorar las situaciones sociales como de diseñar las políticas para abordarlas (Ranci, 2010).

- Por otro lado, frente a la paz social que proporcionó un Estado de bienestar capaz de extender unos mínimos de vida digna para el conjunto de la población, a principios de los ańos ochenta del pasado siglo se produjo lo que algunos autores llamaron «el redescubrimiento de la pobreza» (Gorz, 1998; Bauman, 2005). Las ricas sociedades occidentales, que parecían haber alcanzado el cielo, encontraron el infierno en las calles y las plazas de sus principales ciudades: un número creciente de personas deambulaban sin hogar, sin familia, sin recursos de ningún tipo. Eran personas que ya no estaban en la parte de debajo, sino que habían quedado fuera. Unos nuevos pobres — excluidos- que ya no tenían espacio en la sociedad, ni arriba ni abajo. Aparece así un nuevo eje de desigualdad entre los que forman parte y los excluidos, entre los in y los out (Percy-Smith, 2000; Bauman, 2004).

El breve relato esbozado hasta aquí nos permite construir el marco analítico que utilizaremos para estudiar e interpretar un caso concreto, un marco analítico donde se establecen las tres dimensiones necesarias para abordar la exclusión social en toda su complejidad.

\section{La exclusión como situación}

Desde esta perspectiva, una situación de exclusión se definiría como un estado de necesidad intensa y multifactorial (Hulme et al., 2001). Tanto la intensidad como la multifactorialidad dificultan las respuestas tecnocráticas y sectorializadas típicas del Estado de bienestar y, por lo tanto, exigen respuestas que desbordan las clásicas intervenciones reactivas de sus políticas sociales. Las personas en situación de exclusión se encuentran en una especie de vía muerta; viven desconectadas y sin acceso a unas mínimas condiciones materiales de vida de manera crónica. Son, usando los términos de Bauman (2004), «residuos humanos».

El concepto de cronificación se relaciona con esta via muerta de la que resultaría imposible salir. Frente a la lógica clásica de los itinerarios de insersión social, dominantes durante los años del Estado de bienestar, la cronificación implica quedar atrapado en la última red de protección social, sin posibilidades de incorporarse a un comunidad social de forma normalizada (Moreno, 2000). Las referencias conceptuales a este nuevo fenómeno han sido frecuentes durante los últimos ańos, aunque seguimos sin tener muy claro cómo medirla. En cualquier caso, basándonos en la literatura disponible, en la parte empírica observaremos diversas variables como la acumulación de necesidades, el 
uso que realizan de los servicios sociales o las trayectoria y situaciones laborales (Hulme et al., 2001; Hulme y Shepherd, 2003; Calvo y Dercon, 2009). También nos fijaremos en cómo estas situaciones cronificadas de pobreza severa se relacionan con los orígenes, la soledad o la situación habitacional de las personas que las padecen. Finalmente, en relación con las intervenciones sociales dirigidas a estos colectivos, incorporaremos algunos debates sobre la dignificación, la condicionalidad y el reconocimiento.

\section{La exclusión como riesgo}

La exclusión no es únicamente una intensificación y una acumulación de déficits, sino también un contexto donde se corre el riesgo de deslizarse hacia la exclusión social. Una fragilidad que se ha ido expandiendo y que afecta a un número creciente de personas. Muchas son las razones que explican el riesgo de exclusión, entre las que destacan la precariedad laboral, los déficits relacionales (la soledad) o los límites formativos (Oosterlynck et al., 2019). En cualquier caso, mientras que las situaciones de exclusión se abordan con intervenciones reactivas, y centradas en ocasiones solo en la lucha contra el desempleo, la vulnerabilidad reclama políticas anticipativas —inéditas en el ámbito de las intervenciones sociales y orientadas a las múltiples dimensiones que entran en juego en los procesos de exclusión (Martínez Virto et al., 2019)—.

Así pues, en el apartado empírico intentaremos calcular el grado de vulnerabilidad a partir de las necesidades selectivas, las situaciones laborales, la intesidad de las redes relacionales, los niveles formativos y la salud. Observamos, pues, la multidimensionalidad de la vulnerabilidad y la complejidad de identificarla en un territorio. Podemos anticipar, en cualquier caso, cómo esta complejidad se manifiesta en un territorio donde durante la última década ha visto cómo, simultáneamente, se reducían las tasas de paro y aumentaban las demandas de ayudas sociales.

\section{La exclusión como proceso}

La exclusión, como hemos visto, es una situación y un riesgo, pero también el resultado de un proceso, de unas dinámicas socioeconómicas que generan pautas de acumulación y desigualdad (Stiglitz, 2013). El modelo de crecimiento actúa como una locomotora y, según el ritmo que pretendamos imprimirle, estaremos alejando a los excluidos y generando inestabilidad en los que se encuentran en riesgo de exclusión (Piketty, 2014). De este modo, además de las políticas reactivas y anticipativas ya mencionadas, la exclusión también reclama planteamientos estratégicos que afectan al modelo de desarrollo de nuestras comunidades.

De las tres dimensiones de análisis propuestas, se deduce la necesidad de repensar el contenido tanto de nuestros diagnósticos como de nuestras intervenciones en el ámbito de la exclusión social. Una necesidad derivada de la propia complejidad que acompańa al término exclusión y que, para los efectos de este artículo, vamos a centrar 
en la cronificación y la vulnerabilidad. Estas dos características nos ayudan a entender las problemáticas sociales actuales $\mathrm{y}$, además, suponen un importante desafío tanto para la propia concepción de la política social como para las herramientas habituales de implementación de políticas de inclusión.

\section{ESTUDIO DE CASO: EXCLUSIÓN SOCIAL EN LA COMARCA DE LA GARROTXA}

En este tercer apartado presentaremos el estudio de caso que nos ha de permitir dotar de contenido empírico al marco conceptual diseñado en el apartado anterior. Este estudio se realizó durante los años 2017 y 2018 en La Garrotxa, una región del nordeste de España, como resultado de un convenio de colaboración entre los servicios sociales y la Universidad de Girona (Brugué et al., 2017).

\section{Metodología}

Disponer de un estudio empírico exhaustivo que pueda ofrecer un diagnóstico detallado de la situación de la exclusión social en un territorio concreto no es una tarea sencilla. Se requieren datos muy diversos, de distinta naturaleza y a menudo inexistentes a nivel desagregado. Frente a estas dificultades, se optó por combinar diferentes metodologías:

- En primer lugar, realizamos tres sesiones de trabajo previas a la investigación con una duración de cuatro horas cada una de ellas. En las dos primeras, las personas convocadas eran los miembros de la Mesa de la Pobreza de la Garrotxa ${ }^{1}$, mientras que en la tercera participaron unos treinta profesionales de diferentes ámbitos de la intervención social.

- En segundo lugar, revisamos algunos documentos oficiales (memorias de actuación de instituciones y entidades, Observatorio del Trabajo de la Generalitat de Catalunya, Instituto de Estadística de Catalunya, etc.), con el fin de caracterizar la comarca en términos demográficos, sociales, económicos y laborales.

- En tercer lugar, para obtener datos estadísticamente significativos que no existían a nivel desagregado, diseñamos y ejecutamos una encuesta para el universo de 28773 personas mayores de dieciséis años de la ciudad de Olot $^{2}$. Debido a

1. La Mesa de la Pobreza — un espacio de encuentro, debate y seguimiento de la exclusión social en la comarca- está formada por representantes de los servicios sociales (Consorci d'Acció Social de la Garrotxa), la Agencia de Innovación y Desarrollo Económico (DINAMIG), el Área de Salud Pública, el Instituto Municipal de Educación y Juventud (IME), del DIPSALUT (Diputación de Girona), Caritas, Cruz Roja y la entidad social Mans per a l'Acció Solidària.

2. La encuesta, con un nivel de confianza del $95,5 \%$ y un margen de error de $+/-5 \%$, se realizó para una muestra de 402 personas con cuotas de edad, sexo y nacionalidad. 
problemas técnicos y logísticos, la población de referencia no era la comarca (con datos de 2016, 55998 habitantes repartidos entre 21 municipios), sino la ciudad central, Olot (34000 habitantes, que representan el 60,64\% del total de la comarca). Los datos concretos sobre cronicidad y vulnerabilidad que presentaremos se refieren específicamente a esta ciudad ${ }^{3}$.

- En cuarto lugar, se realizaron entrevistas semiestructuradas para obtener información cualitativa sobre la exclusión social en la comarca ${ }^{4}$. Se entrevistó a un total de diecisiete personas distribuidas entre cuatro perfiles: seis representantes de los diferentes grupos políticos, cuatro profesionales (salud, educación, trabajo social y promoción económica y formación ocupacional), cuatro representantes de entidades de la sociedad civil (empresariales, vecinales, dedicadas al ocio y solidarias) y 3 usuarios de los servicios sociales. En el anexo 1 se puede consultar una tabla con los perfiles, la descripción de las personas y la codificación que hemos utilizado para citarlos en la parte de análisis y discusión.

A partir de este arsenal metodológico, disponemos hoy de un conocimiento detallado de la exclusión social en la Garrotxa. A continuación, escogemos una parte del conocimiento adquirido para ver cómo, en términos de cronicidad y vulnerabilidad social, pasamos de la teoría a la práctica.

\section{Cronificación y vulnerabilidad: el caso de Olot (Garrotxa, Girona)}

a) Una mirada panorámica a las dinámicas que refuerzan el riesgo de pobreza y exclusión social

En el contexto europeo, Cataluña es una región económicamente dinámica, con un PIB por encima de la media y con un ritmo de crecimiento del $2,6 \%$ en $2018^{5}$, por lo que supera a países como Alemania $(1,4 \%)$, Francia $(1,7 \%)$, Italia $(0,9 \%)$ o

3. No detallaremos todas las preguntas de una encuesta que duraba unos veinticinco minutos por persona, ya que solo usaremos aquellas que nos sirven para los objetivos específicos de este artículo. En cualquier caso, las preguntas estaban organizadas en seis bloques: (1) características personales; (2) relación con el municipio (llegada, vivienda, etc.); (3) redes sociales, salud y percepciones en relación a la inmigración; (4) situaciones de exclusión y contactos con los diversos servicios sociales; (5) trabajo, ocupación y rentas, y (6) satisfacción con el presente y expectativas de futuro.

4. Las entrevistas semiestructuradas, como sabemos, se organizan alrededor de unos ejes generales que organizan la conversación. En nuestro caso utilizamos cuatro grandes temas para conducir las entrevistas: (1) diagnóstico de la exclusión social en la comarca; (2) descripción y valoración de las actuaciones que se realizan para convertir la Garrotxa en un territorio inclusivo, (3) valoración de los resultado alcanzados y propuestas de cambio, y (4) expectativas de futuro.

5. Los datos que se analizan a continuación provienen de la base de datos del IDESCAT a nivel catalán y del Eurostat a escala europea. 
Dinamarca (1,5\%). También la actividad industrial y los niveles de competitividad son elevados en relación al entorno europeo. Sin embargo, como se refleja en la tabla 1, los datos muestran la menor capacidad de su modelo de bienestar a la hora de resistir los impactos sociales de la recesión económica y, por lo tanto, de proteger a la población más vulnerable de los devastadores efectos de la crisis.

TABLA 1.

INDiCADORES DE POBREZA Y EXClusión. CATALUÑa, 2007 Y 2020

\begin{tabular}{lcccc}
\hline Indicadores de pobreza y exclusión & $\mathbf{2 0 0 7}$ & $\mathbf{2 0 1 8}$ & $\mathbf{2 0 1 9}$ & $\mathbf{2 0 2 0}$ \\
\hline Población que vive bajo el umbral de la pobreza & $18,2 \%$ & $21,3 \%$ & $19,5 \%$ & $21,7 \%$ \\
\hline $\begin{array}{l}\text { Población que sufre situaciones de privación material } \\
\text { severa (renta inferior al 60\% de la media) }\end{array}$ & $2,5 \%$ & $6,5 \%$ & $5,7 \%$ & $6,2 \%$ \\
\hline $\begin{array}{l}\text { Población que vive en hogares con intensidad de trabajo } \\
\text { muy bajo }\end{array}$ & $4,5 \%$ & $5,8 \%$ & $8,3 \%$ & $9,8 \%$ \\
\hline
\end{tabular}

Fuente: Idescat. Encuesta de condiciones de vida (2021).

Si nos centramos en la Garrotxa, podemos observar cómo las desigualdades de renta son similares a las del conjunto de Cataluña (tabla 2). En términos de desigualdad de rentas y de rentas por debajo del $60 \%$ de la mediana, la situación de la Garrotxa es algo mejor, aunque la situación es ligeramente más negativa cuando nos fijamos en la brecha de las rentas más bajas.

TABLA 2.

INDICADORES DE RENDA MONETARIA. ÁREAS BÁSICAS DE SERVICIOS SOCIALES. CATALUÑ́A, 20 I 2

\begin{tabular}{lccc}
\hline & $\begin{array}{c}\text { Desigualdad de renda } \\
\text { según fuentes } \\
\text { tributarias }\end{array}$ & $\begin{array}{c}\text { Porcentage de rendas } \\
\text { inferiores al 60\% } \\
\text { de la mediana }\end{array}$ & $\begin{array}{c}\text { Brecha de las rendas } \\
\text { inferiores al 60 \% } \\
\text { de la mediana }\end{array}$ \\
\hline Garrotxa (sin Olot) & 45,2 & 27,5 & 38,2 \\
\hline Olot & 46,1 & 27,9 & 38,6 \\
\hline Cataluńa & 49,2 & 31,2 & 37,9 \\
\hline
\end{tabular}

Fuente: elaboración propia a partir de datos de IDESCAT y la Memoria de los Servicios Sociales de la Garrotxa, CASG (2016).

La Garrotxa ${ }^{6}$, además, presenta algunas peculiaridades demográficas y socioeconómicas. Se trata de un territorio donde el crecimiento demográfico entre el 2000 y el 2015 ha mantenido niveles $(18 \%)$ similares a la media catalana (19,9\%); el procentaje de inmigración es del 18,4\%; un $57 \%$ de la población ha nacido en la propia comarca, y se trata de una comarca envejecida, donde la población mayor de 85 años alcanza el 4,1\%. En términos económicos, nos encontramos en un territorio activo

6. Los datos comarcales que se analizan en este párrafo provienen de la base de datos del Observatorio Económico, Social y Medioambiental de la Garrotxa. 
donde, por ejemplo, el porcentaje dedicado a la industria alcanza un destacable $21 \%$ y donde la población activa llega al 62,9\%.

A continuación, ya con el foco situado exclusivamente en la ciudad de Olot, usaremos el trabajo de campo para abordar las dos temáticas centrales de este artículo: la cronificación de la pobreza y la creciente vulnerabilidad de una población en riesgo de exclusión.

b) La pobreza cronificada en Olot: gestionar el presente sin construir el futuro

En la tabla 3 contrastamos unos porcentajes relativamente altos de personas que no pueden permitirse disfrutar de una semana de vacaciones $(35,4 \%)$ o que no pueden hacer frente a un gasto imprevisto de 700 euros $(33,58 \%)$, con porcentajes mucho más bajos de personas que no pueden comer carne o pescado cada dos días $(2,9 \%)$, que no pueden acceder a una lavadora $(3,7 \%)$ o que no pueden mantener su vivienda con una temperatura adecuada en invierno $(5,47 \%)$. También el disponer de un coche es ya imposible para un $16,9 \%$ de la población.

TABLA 3.

Personas Que no PUEDEN ACCEDER A DETERMINAdos BIENES y SERVICIOS. Olot, 20 I 7

\begin{tabular}{lc}
\hline Vacaciones al menos una semana al año & $35,41 \%$ \\
\hline Una comida de carne o pescado al menos cada dos días & $2,99 \%$ \\
\hline Mantener la vivienda a una temperatura adecuada durante el invierno & $5,47 \%$ \\
\hline Un televisor & $3,74 \%$ \\
\hline Una lavadora & $3,74 \%$ \\
\hline Un coche & $16,96 \%$ \\
\hline $\begin{array}{l}\text { No podar hacer frente a gastos básicos de vivienda y suministros durante los } \\
\text { útimos 12 meses }\end{array}$ & $11,72 \%$ \\
\hline Hacer frente a un gasto imprevisto de 700 euros con recursos propios & $33,58 \%$ \\
\hline
\end{tabular}

Fuente: elaboración propia.

Estos datos no suponen, en realidad, ninguna sorpresa. Ya en los grupos de discusión previos a la realización de la encuesta se ponía el acento en la intensificación de las dinámicas de exclusión experimentada durante los últimos años. En palabras de un responsable político: «Desde hace unos años vamos hacía una fractura, aumenta la polarización y disminuye la clase media. A la gente cada vez le cuesta más desenvolverse, cada vez hay más diferencias, y cada vez hay menos derechos y menos servicios. Y además, parece que la mentalidad de la gente ha ido cambiando, hasta el punto de aceptarlo. Una situación muy grave y muy silenciada» (E1PP) ${ }^{7}$.

7. En el anexo 1 presentamos la tabla de personas entrevistadas y la codificación utilizada para identificar el perfil y la decripción de las personas. De esta manera se puede consultar a quién corresponden las citas escogidas de las entrevistas y los grupos de discusión. 
Por otra parte, la intensidad de la exclusión —y también las posibilidades de cronificación - aumenta cuando se acumulan las carencias y las dificultades de acceso a bienes y servicios básicos. En este sentido, según datos de nuestra encuesta, el porcentaje de personas que sufre al menos cuatro de las siete carencias planteadas en las preguntas $\mathrm{AROPE}^{8}$ alcanza el 4,73\%. Estaríamos ante el núcleo duro de la exclusión social en Olot. De nuevo, este porcentaje coincidiría con las estimaciones subjetivas que aparecieron en los grupos de trabajo y en algunas entrevistas con profesionales: «Las personas en situación de exclusión social representan un volumen más reducido que en otras comarcas, aunque el número ha ido incrementándose de manera progresiva durante la última década. Deben estar entre el 5 y el $10 \%$.» (E3P).

Una franja algo más amplia, que alcanzaría el $10 \%$, coincidiría, otra vez según datos de la encuesta, con el porcentaje de personas que afirman haber utilizado los servicios sociales en algún momento de su vida. También coincide con la respuesta a la pregunta sobre si ha tenido dificultades para hacer frente a gastos básicos como el alquiler, la hipoteca o los suministros (11,72\%).

Completando los datos con las aportaciones cualitativas, en Olot existe aproximadamente un $10 \%$ de personas que recurren a ayudas de los servicios sociales. Al mismo tiempo, los profesionales sostienen que este $10 \%$ se reparte entre un $5 \%$ que son usuarios habituales y un $5 \%$ que son usuarios puntuales. Los primeros formarían un colectivo crónico de personas dependientes y con escasas —o nulas - posibilidades de superar esta situación, mientras que el otro $5 \%$ estaría en una situación delicada, pero sin haber atravesado todavía la frontera de la cronificación.

Una vez delimitado este núcleo de pobreza severa y, en muchos casos, cronificada, nos interesa saber quiénes son las personas en esta situación, cuáles son sus características, sus perfiles y sus trayectorias. Las tres variables que mejor correlacionan con las dificultades para hacer frente a los pagos básicos $(11,72 \%)$ son la nacionalidad (tabla 4), la proximidad familiar (tabla 5) y la situación habitacional (tabla 6).

TABLA 4.

Dificultades para REALIZAR Los pagos básicos SEgÚn NACIONALIDAd (N. ${ }^{\circ}$ abSOlUto PERSONAS, N. ${ }^{\circ}$ ABSOLUTO QUE SE ESPERARÍA EN CASO DE NO RELACIÓN Y \% COLUMNA)

\begin{tabular}{|c|c|c|c|}
\hline & Española & Extranjera & Total \\
\hline \multirow{3}{*}{ Sí (sufren dificultades) } & 23 & 24 & 47 \\
\hline & $(38,6)$ & $(8,4)$ & $(47)$ \\
\hline & $7,0 \%$ & $33,3 \%$ & $11,7 \%$ \\
\hline
\end{tabular}

8. La tasa AROPE (at risk of poverty andlor exclusion) es un indicador creado por la Red Europea de Lucha contra la Pobreza que se encarga de medir la población en riesgo de exclusión social y pobreza según se cumpla uno de los tres criterios siguientes: vivir en un hogar con una renta inferior al umbral de la pobreza (valor que corresponde al $60 \%$ de la mediana de la renta nacional equivalente en unidades de consumo); estar en situación de privación de material severa, o vivir en un hogar con baja intensidad de trabajo. 


\begin{tabular}{lccc}
$\ldots / \ldots$ & & & \\
\hline & Espańola & Extranjera & Total \\
\hline \multirow{3}{*}{ No (sufren dificultades) } & 306 & 48 & 354 \\
& $(209,4)$ & $(63,6)$ & $(354)$ \\
& $93,0 \%$ & $66,7 \%$ & $88,3 \%$ \\
Total & 329 & 72 & 401 \\
& $(329)$ & $(72)$ & $(401)$ \\
& $100 \%$ & $100 \%$ & $100 \%$ \\
\hline
\end{tabular}

Fuente: elaboración propia.

TABLA 5.

Dificultades PARA REALIZAR LOS PAGOS BÁSICOS SEGÚN PROXIMIDAD DE LOS VÍNCULOS FAMILiARES (.$^{\circ}$ ABSOlUTO DE PERSONAS, N. $^{\circ}$ ABSOLUTO QUE SE ESPERARÍA EN CASO DE NO RELACiÓN Y \% COLUMNA)

\begin{tabular}{lcccccccc}
\hline & $\begin{array}{c}\text { Mismo } \\
\text { municipio }\end{array}$ & $\begin{array}{c}\text { Otro } \\
\text { municipio } \\
\text { Garrotxa }\end{array}$ & $\begin{array}{c}\text { Otro } \\
\text { municipio } \\
\text { Girona }\end{array}$ & $\begin{array}{c}\text { Otro } \\
\text { municipio } \\
\text { Cataluña }\end{array}$ & $\begin{array}{c}\text { Otro } \\
\text { municipio } \\
\text { España }\end{array}$ & $\begin{array}{c}\text { Otro } \\
\text { municipio } \\
\text { extranjero }\end{array}$ & $\begin{array}{c}\text { Sin } \\
\text { familia } \\
\text { referente }\end{array}$ & Total \\
\hline \multirow{3}{*}{ Sí } & 18 & 0 & 10 & 3 & 2 & 12 & 2 & 47 \\
& $(9,2)$ & $(4,9)$ & $(4,6)$ & $(3,3)$ & $(1,2)$ & $(3,3)$ & $(0,6)$ & $(47)$ \\
& $7,2 \%$ & $0,0 \%$ & $25,6 \%$ & $10,7 \%$ & $20,0 \%$ & $42,9 \%$ & $40,0 \%$ & $11,7 \%$ \\
\hline \multirow{3}{*}{ No } & 231 & 42 & 29 & 25 & 8 & 16 & 3 & 354 \\
& $(219,8)$ & $(37,1)$ & $(34,4)$ & $(24,7)$ & $(8,8)$ & $(24,7)$ & $(4,4)$ & $(354)$ \\
& $92,8 \%$ & $100 \%$ & $74,4 \%$ & $89,3 \%$ & $80,0 \%$ & $57,1 \%$ & $60,0 \%$ & $88,3 \%$ \\
\hline \multirow{2}{*}{ Total } & 249 & 42 & 39 & 28 & 10 & 28 & 5 & 401 \\
& $(249)$ & $(42)$ & $(39)$ & $(28)$ & $(10)$ & $(28)$ & $(5)$ & $(401)$ \\
& $100 \%$ & $100 \%$ & $100 \%$ & $100 \%$ & $100 \%$ & $100 \%$ & $100 \%$ & $100 \%$ \\
\hline
\end{tabular}

Fuente: elaboración propia.

TABLA 6.

Dificultades PARA REAlizar los PAGOS BÁSICOS SEGÚN SITUACIÓN HABITACIONAL (N. ${ }^{\circ}$ ABSOLUTO PERSONAS, N. ${ }^{\circ}$ ABSOlUto QUE SE ESPERARÍA EN CASO DE NO RELACión Y \% COLUMNa)

\begin{tabular}{cccccc}
\hline & $\begin{array}{c}\text { Propiedad pagando } \\
\text { hipoteca }\end{array}$ & $\begin{array}{c}\text { Propiedad con } \\
\text { hipoteca pagada }\end{array}$ & $\begin{array}{c}\text { Propiedad por herencia } \\
\text { o donación }\end{array}$ & Alquiler & Total \\
\hline \multirow{2}{*}{ Sí } & 7 & 3 & 1 & 36 & 47 \\
& $(9,6)$ & $(20,6)$ & $(3,3)$ & $(13,5)$ & $(47)$ \\
& $8,5 \%$ & $1,7 \%$ & $3,6 \%$ & $31,3 \%$ & $11,7 \%$ \\
\hline \multirow{4}{*}{ No } & 75 & 173 & 27 & 79 & 354 \\
& $(72,4)$ & $(155,4)$ & $(24,7)$ & $(101,5)$ & $(354)$ \\
& $91,5 \%$ & $98,3 \%$ & $96,4 \%$ & $68,7 \%$ & $88,3 \%$ \\
\hline \multirow{2}{*}{ Total } & 82 & 176 & 28 & 115 & 401 \\
& $(82)$ & $(176)$ & $(28)$ & $(115)$ & $(401)$ \\
& $100 \%$ & $100 \%$ & $100 \%$ & $100 \%$ & $100 \%$ \\
\hline
\end{tabular}

Fuente: elaboración propia. 
Con datos estadísticamente significativos, la población extranjera, aquellos que tiene menos proximidad familiar y aquellos que están de alquiler son más propensos a padecer situaciones de exclusión social severas y tendentes a la cronificación.

También el mercado laboral tiene relación con la cronificación de la pobreza, pues se trata de la única salida que se ha planteado tradicionalmente a las situaciones de exclusión. Una salida hoy taponada para muchas personas que probablemente ni van a encontrar trabajo ni, si lo encontraran, les proporcionaría los recursos mínimos para su subsistencia. En el año 2018, la tasa de paro en Olot se situaba por debajo del $10 \%$. Sin embargo, con datos de nuestra encuesta, más del $40 \%$ de los parados llevaban más de un año buscando trabajo sin ningún éxito y más de un $70 \%$ no han concertado ninguna entrevista laboral durante los últimos tres meses. Se trata, por lo tanto, de un colectivo relativamente reducido, pero con tendencia a la cronificación.

Nos encontramos, pues, ante un colectivo de personas (entre el $5 \%$ y el $10 \%$ de la población de Olot) que se encuentran en una situación de exclusión severa y que comparte un perfil caracterizado por el origen extranjero, la distancia respecto las redes familiares, una situación habitacional inestable y dificultades laborales graves. Al mismo tiempo, los datos de la tabla 7 muestran que un $44,7 \%$ de las personas en esta situación afirma no recibir ningún tipo de ayuda. Entre los que sí reconocen recibir algún tipo de ayuda, el $31,9 \%$ se refiere a los familiares y el 10,6\% a relaciones de amistad. Estas redes informales, por lo tanto, son percibidas como una fuente relevante de soporte. La presencia de las instituciones públicas y de las entidades sociales es escasa. La soledad y el aislamiento aparecen como características que acompañan a la exclusión social.

TABLA 7.

Ayudas PARA HaCER FRENTE A LAS NECESIDADES BÁSICAS

\begin{tabular}{lcc}
\hline & Frecuencia & $\%$ \\
\hline Nadie & 21 & $44,7 \%$ \\
\hline La familia & 15 & $31,9 \%$ \\
\hline El ayuntamiento & 7 & $14,9 \%$ \\
\hline Los amigos & 5 & $10,6 \%$ \\
\hline Otras administraciones públicas & 5 & $10,6 \%$ \\
\hline Entidades sociales / asociaciones no lucrativas & 1 & $2,1 \%$ \\
\hline
\end{tabular}

Fuente: elaboración propia a partir de los resultados de la encuesta.

Resulta relevante subrayar cómo una parte importante de este colectivo manifiesta no recibir ayuda de ningún tipo. El resultado — tal como se nos explica en las entrevistas - es una combinación de conformismo y desesperación: acaban aceptando la inevitabilidad de su situación y su impotencia para superarla, dando así una definición percibida del concepto teórico de cronificación: «Todo en conjunto ha hecho que las personas nos conformemos con lo que tenemos. Frente a la falta de recursos y de 
ayudas, las personas terminamos siendo conformistas y aceptando nuestra situación, sin querer mejorar» (E2S).

El núcleo duro de la pobreza padece, de ese modo, una situación de cronificación que, en los grupos de discusión fue descrita como: «No solo por sus necesidades de presente sino, sobre todo, por su ausencia de futuro» (E3P).

Una situación que, además, se vive en solitario y se experimenta como un fracaso personal, les hace invisibles y lastima gravemente su autoestima. Y frente a esta ecuación perversa de soledad-fracaso-invisibilidad-baja autoestima, la respuesta se concentra en los aspectos más superficiales, en las urgencias. Así lo explicaba una profesional del ámbito social: «Las instituciones vamos tarde y solo tapamos agujeros [...]. Se atienden los casos a partir de respuestas de emergencia en temas como la vivienda o los suministros; pero son respuestas meramente asistenciales que nunca van a la raíz del problema. Se mantienen, así, las situaciones de fragilidad, de sufrimiento y, sobre todo, de injusticia social» (E2P).

Finalmente, de la información obtenida a través de entrevistas y grupos de discusión, las profesionales de los servicios sociales se sienten frustradas ante la incapacidad de pasar de unas intervenciones «que alivian el presente a otras que no logran construir el futuro» (E3P).

Unos límites que, además, se traducen en un trato indigno a las personas que se encuentran en esta situación. Se percibe la necesidad de una reorientación radical, aunque desconocen cómo abordarla: «Es necesario replantearlo todo a fondo. Hemos de conseguir trabajar para promover un cambio que transforme la vida de las personas, en vez de limitarnos a ofrecer intervenciones paliativas» (E4PP).

c) La vulnerabilidad en Olot: ya todos podemos caer

El riesgo y la vulnerabilidad suponen unos intangibles y, al mismo tiempo, pueden presentarse a partir de un amplio abanico de grados. No se trata, por lo tanto, de una realidad sencilla ni de observar ni de calcular. En el caso de Olot, un $34 \%$ de los encuestados responden negativamente a tres de las siete situaciones que plantean los interrogantes AROPE. ¿ Son estas las personas vulnerables que están en riesgo de exclusión? Una pregunta difícil de responder, aunque nos sitúa en la pista de una población que quizá todavía no se encuentra en una situación de exclusión pero que podría estar acercándose a ella.

En los grupos de discusión y en las entrevistas hay referencias constantes a la creciente difusión del riesgo de exclusión. No sabemos cómo definirlo con exactitud, y todavía menos cómo calcularlo, pero hay un amplio reconocimiento sobre su existencia. En palabras de los entrevistados, esta realidad está provocando un temor difuso y generalizado: «Se ha instalado la incertidumbre en la vida cuotidiana de las personas. La gente tiene menos dinero e incrementa su miedo a caer en situaciones de pobreza. El problema es que éste miedo se está generalizando» (E4S). "Hemos perdido las clases medias que nos hacían de puente. Los ricos son más ricos, los pobres más pobres, y la clase media se va acercando cada vez más a los pobres. (E3BSS)». 
Al mismo tiempo, hemos observado lo complicado que es identificar el perfil de esta población vulnerable. Un ejercicio tentativo consiste en cruzar la variable capacidad de las personas para afrontar un gasto de setecientos euros con algunas variables de carácter personal. Aparece una relación significativa entre la capacidad de afrontar este gasto y el número de ańos que la persona lleva viviendo en el municipio. También aparece una relación significativa, ahora negativa, con las personas que viven solas o las familias monoparentales y que disponen de redes familiares más débiles y distantes. Tres variables —arraigo, soledad y soporte comunitario— que, por lo tanto, se asocian tanto con la exclusión como con la vulnerabilidad.

En cualquier caso, a la hora de diseñar políticas es muy relevante saber qué explica la vulnerabilidad. ¿Cuáles son los factores que explican el riesgo de exclusión y que, por lo tanto, deberían ser objeto de intervención? En este sentido, partiendo de consideraciones teóricas, hemos identificado y analizado cuatro factores de vulnerabilidad: la salud, la situación laboral, las redes sociales y familiares, y el origen.

La salud es un primer factor para explicar la vulnerabilidad. Según los datos de nuestra encuesta, un 49,25\% de la población de Olot considera que su estado de salud es bueno y un $25,37 \%$ que es muy bueno. Al mismo tiempo, un $3 \%$ considera que su estado de salud es malo o muy malo, y un $21,64 \%$ define su estado de salud como regular. También es reseñable, como subrayaba una de las personas entrevistadas, la importancia de los problemas de salud mental relacionados con las situaciones de estrés que comportan las situaciones de vulnerabilidad: «Hay mucha gente que no ve ningún futuro, no ve ninguna salida. Esto provoca depresiones, problemas de salud, afectación de la vida social y familiar» (E3S).

Adicionalmente, solo un 4,75\% de los entrevistados reconocen necesitar de algún soporte relacionado con su estado de salud. Un dato que nos podría indicar que solo los que manifiestan explícitamente un mal estado de salud piden ayuda.

Un segundo factor especialmente relevante para explicar la vulnerabilidad es el laboral, pues se trata del único factor que tradicionalmente ha sido considerado como la palanca hacia la inclusión. En relación con la situación laboral, los datos de la encuesta muestran una amplia variedad de situaciones. Tal como se recoge en la tabla 8 , el índice de personas trabajando es elevado, por encima del 50\%. También el porcentaje de jubilados cobrando una pensión, que representa más del $25 \%$ de la población de Olot. El colectivo de personas que busca trabajo y no ingresa ninguna prestación por desempleo vuelve a acercarse a la cifra del $5 \%$.

TABLA 8.

¿CuÁl de las Siguientes SITUACiONES LABORALES ES LA SUYa?

\begin{tabular}{lc}
\hline Trabaja & $52,49 \%$ \\
\hline Estudia & $5,22 \%$ \\
\hline Trabaja y estudia & $2,99 \%$ \\
\hline Busca trabajo y cobra del paro & $2,99 \%$ \\
\hline
\end{tabular}




\begin{tabular}{lc}
$\ldots . \ldots$ & \\
\hline Busca trabajo y no cobra del paro & $4,48 \%$ \\
\hline Tareas del hogar & $2,24 \%$ \\
\hline Jubilado cobrando pensión & $25,62 \%$ \\
\hline Jubilado sin pensión & $1,24 \%$ \\
\hline No trabaja ni estudia ni busca trabajo & $0,5 \%$ \\
\hline Invalidez por discapacidad & $2,24 \%$ \\
\hline
\end{tabular}

Fuente: elaboración propia a partir de los resultados de la encuesta.

En el caso de la Garrotxa ya habíamos anticipado que, a pesar de tratarse de una comarca con índices de ocupación por encima de la media catalana (un 62,94\% de los encuestados se declaran laboralmente activos), genera también situaciones de exclusión laboral (a través de la cronificación en el paro de una parte relativamente pequeña de la población) y de vulnerabilidad laboral, entendiendo que sus salarios medianos son inferiores a la media y que, por lo tanto, la capacidad para resistir determinadas contingencias puede ser menor. De hecho, en las entrevistas con profesionales y en los grupos de discusión se constató la creciente preocupación en la comarca por esta incipiente categoría de los llamados «trabajadores pobres» (Standing, 2011).

Estas percepciones tienen su reflejo en las cifras. Para empezar, si al 62,94\% de población laboralmente activa le sumamos el $25,82 \%$ que se declaran como jubilados que cobran una pensión, nos acercaríamos a un $90 \%$ de personas en una situación estable desde la perspectiva de los ingresos. $\mathrm{O}$, dicho en otros términos, poco más de un $10 \%$ de población $(11,44 \%)$ se encuentra en situaciones de exclusión o vulnerabilidad severa.

Por otra parte, podemos concentrar nuestra atención en aquellos que se manifiestan laboralmente activos, entre los cuales se puede distinguir entre trabajadores asalariados $(85,4 \%)$, empresarios o autónomos sin trabajadores $(10,36 \%)$ y autónomos con trabajadores $(4,5 \%)$. Los autónomos sin trabajadores representan un colectivo diverso, pero donde son frecuentes la situaciones de vulnerabilidad. Al mismo tiempo, entre los trabajadores asalariados podemos distinguir aquellos que disponen de contratos indefinidos (74,07\%), funcionarios públicos $(7,41 \%)$ y aquellos que están contratados temporalmente (17,5\%, entre los cuales un 48,5\% tienen contratos de menos de seis meses). Este $17,5 \%$ podría estar representando un nuevo espacio de vulnerabilidad que, sumado al $11,44 \%$ que ya se encontraría fuera del mercado laboral, situaría potencialmente a un tercio de la población en una situación de vulnerabilidad laboral.

Un tercer indicador desde el cual calcular la población laboralmente vulnerable en Olot — siempre en función de hipótesis que nos acercan a una realidad de contornos muy difusos - sería la estructura salarial. Tal como muestra la tabla 9, los salarios que podríamos considerar de franja alta, por encima de los 1800 euros al mes, no alcanzan el 10\%; mientras que aquellos que se sitúan por debajo de los 1200 euros al mes ascienden al $56,37 \%$. No podemos fijar una franja salarial como límite para determinar situaciones de vulnerabilidad, entre otras cosas porque esto dependerá de otros 
factores como las aportaciones familiares o la situación habitacional. Pero podemos intuir que, con esta estructura salarial, más de la mitad de la población de Olot puede estar en zonas de riesgo o vulnerabilidad.

TABLA 9.

INGRESOS INDIVIDUALES DECLARADOS

\begin{tabular}{lc}
\hline Menos de $655 € / \mathrm{mes}$ & $5,39 \%$ \\
\hline Entre $655-900 € / \mathrm{mes}$ & $18,63 \%$ \\
\hline Entre $900-1200 € / \mathrm{mes}$ & $32,35 \%$ \\
\hline Entre $1200-1500 € / \mathrm{mes}$ & $24,02 \%$ \\
\hline Entre $1500-1800 € / \mathrm{mes}$ & $9,8 \%$ \\
\hline Más de $1800 € / \mathrm{mes}$ & $9,8 \%$ \\
\hline
\end{tabular}

Fuente: elaboración propia a partir de los resultados de la encuesta.

Es complicado identificar los perfiles que ubicamos en estos espacios de vulnerabilidad, especialmente porque la reducción del número de casos sobre los que vamos trabajando hace prácticamente imposible establecer relaciones estadísticamente significativas. En cualquier caso, la vulnerabilidad laboral parece relacionarse de forma especialmente intensa con la edad y la nacionalidad; mientras que esta relación es más débil — cuestionando una hipótesis muy consolidada — con el nivel de estudios. Por precaución estadística no nos atrevemos a avanzar más en esta dirección, pero se abren aquí algunos interrogantes interesantes.

El cuarto factor que, según la literatura, incide en los niveles de vulnerabilidad, hace referencia a la densidad de las redes sociales y familiares. En este sentido, podríamos afirmar que cuando las redes familiares son muy densas, entonces son capaces de ejercer una protección efectiva frente a la vulnerabilidad. Así, un $62 \%$ de los encuestados tienen sus redes familiares en el propio municipio, un $11 \%$ en la comarca de la Garrotxa, un $10 \%$ en la provincia de Girona, un $7 \%$ en Cataluña y un $2,49 \%$ en España. Únicamente un 6,9\% tiene estas redes familiares en el extranjero y un $1,24 \%$ afirma no tener ningún familiar próximo.

Sin embargo, una cosa es disponer de redes familiares próximas y otra es que estas actúen de manera efectiva. En este sentido, la encuesta también preguntaba sobre la frecuencia de estas relaciones: un 53,9\% afirman tener relaciones muy frecuentes y un $28,4 \%$ de manera frecuente. Es decir, el $20 \%$ restante —otra vez potencialmente más vulnerable - reconocería tener relaciones ocasionales $(11,08 \%)$, muy infrecuentes $(5,79 \%)$ o, incluso, no tener ninguna relación $(0,76 \%)$. Aparece un contraste entre una parte importante de la población que cuenta con un soporte familiar potente y de proximidad y una parte menos numerosa - pero significativa - de personas que no contarían con esta red de protección.

Al lado de las redes familiares, se encontraría una red social de asociaciones y entidades que también podrían contribuir a la protección de los más vulnerables. Sin 
embargo, esta situación en nuestra encuesta se muestra frágil. Únicamente un 20,4\% afirma pertenecer a alguna entidad y un 4,7\% estar activamente colaborando en ellas. Tampoco la participación política supera el $17 \%$.

Un último factor, en buena parte relacionado con la falta de redes, es el origen de las personas. El hecho de ser inmigrante se relacionaba significativamente con las situaciones de exclusión más severas, y también parece vincularse con la vulnerabilidad. En este sentido, resulta relevante analizar las percepciones sobre el hecho migratorio en la ciudad de Olot. Aparentemente, como aparece en la tabla 10, estas percepciones no serían excluyentes con la población inmigrada: un 93,74\% de los encuestados se muestran muy de acuerdo-bastante de acuerdo con que necesitamos trabajar para mejorar su integración. También un 89,29\% se declaran muy de acuerdo-bastante de acuerdo con que deberían tener los mismos derechos que el resto de los españoles.

TABLA iO.

PERCEPCIONES EN TORNO A LA POBLACIÓN INMIGRANTE

\begin{tabular}{lcccc}
\hline & $\begin{array}{c}\text { Muy de } \\
\text { acuerdo }\end{array}$ & $\begin{array}{c}\text { Bastante } \\
\text { de acuerdo }\end{array}$ & $\begin{array}{c}\text { Más bien en } \\
\text { desacuerdo }\end{array}$ & $\begin{array}{c}\text { Totalmente } \\
\text { desacuerdo }\end{array}$ \\
\hline Los inmigrantes nos quitan el trabajo & $5,5 \%$ & $15,18 \%$ & $25,92 \%$ & $53,4 \%$ \\
\hline $\begin{array}{l}\text { Reciben un trato de favor por parte } \\
\text { de la administración }\end{array}$ & $17,44 \%$ & $32,15 \%$ & $22,34 \%$ & $28,07 \%$ \\
\hline $\begin{array}{l}\text { Deberían tener los mismos derechos } \\
\text { que los españoles }\end{array}$ & $55,61 \%$ & $33,68 \%$ & $8,88 \%$ & $1,83 \%$ \\
\hline $\begin{array}{l}\text { Empeoran el funcionamientos } \\
\text { de los servicios públicos }\end{array}$ & $6,08 \%$ & $20,9 \%$ & $34,66 \%$ & $38,36 \%$ \\
\hline $\begin{array}{l}\text { Deberían promoverse acciones } \\
\text { para integrarlos en la sociedad }\end{array}$ & $70,5 \%$ & $23,24 \%$ & $5,22 \%$ & $1,04 \%$ \\
\hline
\end{tabular}

Fuente: elaboración propia a partir de la encuesta.

Sin embargo, podemos también observar los datos agrupando a todos aquellos que no muestran una actitud clara al respecto. Es decir, solo un 53,4\% se muestran totalmente en desacuerdo con la afirmación de que «los inmigrantes nos quitan el trabajo», mientras que casi la mitad de los encuestados se muestran muy de acuerdo (5,5\%), bastante de acuerdo (15,18\%) o más bien en desacuerdo (25,92\%) con la misma. Haciendo esta lectura del cuadro, tan solo un $28,07 \%$ están totalmente en desacuerdo con que «los inmigrantes reciben un trato de favor»; un 55,6\% se muestran claramente de acuerdo con que «deberían tener los mismos derechos»; un 38,36\% está totalmente en desacuerdo con que «empeoran los servicios públicos», y un 29,5\% no se muestra contundentemente a favor de "acciones para promover su integración». Estos datos nos hablan de la vulnerabilidad de la población inmigrante. Una población que sufre percepciones negativas que dificultan su integración, aunque estas se expresen a partir de ciertos circunloquios que las hagan políticamente más aceptable. 
En definitiva, podemos concluir este apartado señalando la existencia de un colectivo cada vez más numeroso de personas frágiles a la hora de afrontar posibles situaciones de necesidad. No es fácil delimitar sus contornos estadísticamente. Tampoco definir claramente quienes son ni las razones de su vulnerabilidad. Pero tanto los datos de la encuesta como las aportaciones cualitativas nos han aportado algunas intuiciones interesantes. Una vulnerabilidad que se presenta como algo difuso, pero que tiene un fuerte impacto en la realidad social de la comarca. Tal como lo expresaba una de las profesionales entrevistadas: «Uno de los aspectos mas problemáticos es el aumento de la crispación. Y es que cuando la gente tiene necesidades y no llega a finales de mes se genera un sentimiento de injusticia muy grande» (E3PP).

Frente a esta nueva realidad, los servicios sociales reconocen su desconcierto. De entrada, no saben ni quiénes son las personas vulnerables ni dónde están ni cómo llegar a ellas. Se reclama capacidad para generar conocimiento sobre nuestras comunidades y para diseñar e implementar políticas sociales de carácter preventivo, un terreno en el que los profesionales reconocen su inexperiencia y sus dudas. Existen iniciativas que trabajan el capital humano y el capital comunitario, que podrían ser interpretadas como intentos en esta dirección, aunque, tal como reconocen todas las personas entrevistadas, queda un largo camino por recorrer.

\section{DE LA TEORÍA A LA PRÁCTICA: NUEVOS RETOS DE FUTURO}

Tras nuestra aproximación empírica, podemos afirmar que ha aumentado y se ha generalizado la vulnerabilidad, la exclusión como riesgo. Al mismo tiempo, también hemos observado la aparición de un grupo de personas sin posibilidades reales de superar su situación de exclusión social; es decir, personas para las cuáles la pobreza se ha convertido en una situación crónica, la exclusión como situación. Esta doble constación empírica supone desafíos a nivel práctico y teórico.

De entrada, hemos podido constatar la utilidad de los conceptos de cronificación y vulnerabilidad a la hora de definir la realidad social empírica de un territorio concreto. Es cierto que la literatura académica todavía tiene que recorrer un largo camino para convertir estos conceptos teóricos en variables que nos permitan hacerlos operativos, de manera que en este artículo hemos pretendido realizar algunas aportaciones. Así pues, combinando datos cuantitativos e informaciones cualitativas, hemos dotado de referencias empíricas a unos conceptos que nos han permitido dibujar con cierto detalle un retrato de la situación social en la ciudad de Olot.

En este sentido, hemos podido identificar un núcleo de entre un $5 \%$ y un $10 \%$ de personas en situación de pobreza severa y con posibilidades muy escasas de superar esta situación. Se trata de un colectivo que utiliza de manera habitual y continuada los servicios sociales, que se encuentra apartado de manera definitiva del mercado laboral y que sufre una acumulación de déficits tanto materiales como relacionales. Identificando este colectivo y esbozando sus rasgos distintivos hemos puesto cara al concepto de cronificación. Por otro lado, también hemos discutido las variables que conforman 
un entorno social donde un porcentaje creciente y significativo de la población de Olot se encuentra en una situación de vulnerabilidad social. Ciertamente, la vulnerabilidad no es un concepto diáfano y que pueda ser definido y medido de manera unívoca. La vulnerabilidad sería una cuestión de grado, de manera que hemos podido identificar diferentes variables y observar diferentes intensidades.

Tal como afirmábamos anteriormente, necesitamos más estudios que intenten traducir los conceptos teóricos en análisis empíricos. Tal como nos comentaba una de las profesionales entrevistadas: «Necesitamos poder explicar con números y evidencias una realidad que a muchos les cuesta entender» (E1P).

En este artículo hemos realizado una contribución en esta dirección, pero también hemos recogido percepciones cualitativas de un buen número de actores que nos permiten identificar algunos retos de futuro desde la perspectiva de las políticas sociales.

Hasta hace poco más de una década, los profesionales de la intervención social conocían a sus usuarios, sabían cómo eran y disponían de un arsenal suficiente de recursos e instrumentos para paliar sus condiciones de pobreza o exclusión. Además, siguiendo una lógica heredada del siglo XIX, dominaba el carácter asistencial de unas políticas que mostraban solidaridad con las personas pobres sin dejar de reconocer que ellas mismas eran, de hecho, las responsables de su situación (Bourdieu, 2000). De hecho, el Estado de bienestar habla hoy el lenguaje de la inclusión social, pero produce el efecto contrario, la exclusión social, a través de medidas cada vez más selectivas y condicionadas que prescriben comportamientos paradójicos a los potenciales beneficiarios (Moruzzi y Prandini, 2020).

De este modo, el primer objetivo de cualquier política social debía ser reconducir estas malas actitudes y comportamientos. Esta perspectiva ha dado forma, históricamente, a unas políticas sociales más interesadas en la lucha contra los pobres que contra la pobreza (Raventós y Wark, 2018). Sus antecedentes, recogidos en el libro de Bauman (2005), los encontramos en las housing poor o en las leyes de vagos y maleantes características del siglo XIX e incluso de las primeras décadas del siglo Xx. Un tipo de planteamiento que implica que la naturaleza de las intervenciones sociales debe ser temporal y que personas en situación de pobreza tienen que aprender a ocuparse de ellas mismas.

Hoy, sin embargo, este tipo de argumento ha perdido mucho sentido, especialmente cuando se extiende la vulnerabilidad y el riesgo de exclusión social. Esta deja de ser una culpa individual y pasa a convertirse en una responsabilidad contextual. Y mientras este contexto no sea transformado, la situación de exclusión puede convertirse en un callejón sin salida, en una situación cronificada (Sennett, 1998). En este sentido, se hace necesario reformular en profundidad tanto los conceptos como las herramientas de las políticas sociales (Hulme et al., 2001; Hulme y Shepherd, 2003; Hickey y Brancking, 2005), y abordar la exclusión también como un proceso.

Además, en nuestras sociedades, junto a un porcentaje creciente pero todavía reducido de personas en situación de exclusión social, observamos un crecimiento exponencial de las personas en situación de vulnerabilidad. Personas que padecen diferentes tipos de fragilidad se deslizan hacia la pendiente de la exclusión (Taylor-Gooby, 2004), un terreno desconocido para unos servicios y unas políticas sociales que se ven obligados a tratar 
personas que no expresan demandas que todavía no tienen. Una tarea preventiva que nunca antes han realizado y que se dirige a un colectivo tan amplio com diversificado. Un colectivo también distante, pues ni se ha acercado a solicitar prestaciones ni, en muchos cosas, es siquiera consciente que puede hacerlo en el futuro (Hulme et al., 2001).

En este nuevo escenario, las políticas sociales deben reforzarse y ampliar su ámbito de acción para lograr, además de paliar las necesidades sociales, prevenir la fractura social y abordar la vulnerabilidad (Martínez et al., 2019). La literatura especializada considera que tendremos que cuestionar la condicionalidad que ha impregnado los servicios sociales hasta la actualidad. No es únicamente un problema instrumental, sino también conceptual, pues se trata de una idea que impregna nuestros marcos culturales. Pero sin esta transformación será imposible abordar la cronicidad de las situaciones de exclusión social. Las garantías universales de ingresos aparecen como una posible solución, pero habrá que convencer al conjunto de la sociedad de que no se producirán efectos perversos.

También deberemos trasladarnos de las certezas y las seguridades vinculadas a la gestión de ayudas, a la construcción de respuestas corales y relacionales en contextos de riesgo e incertidumbre. Especialmente en el terreno desconocido de la vulnerabilidad, no podremos seguir con las viejas inercias. La innovación social se convertirá en un reto que nos obligará tanto a dotarnos de nuevas capacidades para generar conocimiento como de entornos donde experimentar con nuevas propuestas (Ellis y White, 2000; Rodríguez, 2016). Unas propuestas que, además, ya no se enfrentaran solo con casos personales, sino que deberán abordar dinámicas y procesos de exclusión desde una perspectiva multidimensional (Laparra y Pérez, 2008). No podremos seguir batallando con los pobres si no declaramos la guerra a la pobreza.

Finalizamos, pues, este artículo subrayando la importancia de conectar el conocimiento empírico con la reflexión conceptual. Desde la inmersión práctica en un territorio concreto hemos visto cómo se transforma la realidad social, al tiempo que hemos comprobado cómo para entenderla y para hacerle frente también hemos de renovar nuestros marcos teóricos. La actual realidad social exige nuevas formas de conocerla y de pensarla. Conocimiento empírico y reflexión conceptual, dos ingredientes indispensable para diseñar las políticas sociales y construir comunidades cohesionadas en un futuro que ya es presente.

\section{Referencias}

Adelantado, José. (ed.). 2000. Cambios en el Estado de bienestar. Barcelona: Icaria.

Belzunegui, Ángel y Francesc Valls. 2019. Diagnosi social de Catalunya 2018: l'estat de la pobresa. Barcelona: Departament de Treballs, Afers Socials i Famílies.

Bauman, Zygmunt. 2004. Wasted lives: Modernity and its outcasts. Oxford: Polity, Blackwell.

Bauman, Zygmunt. 2005. Work, consumerism and the new poor. Buckingham: Open University Press. 
Beck, Ulrich. 1992. Risk society: Towards a new modernity. Londres: Sage.

Béland, Daniel. 2007. "The social exclusion discourse: Ideas and policy change», Policy and Politics, 35 (1): 123-139. Disponible en: https://doi.org/10.1332/0305 57307779657757.

Bourdieu, Pierre. 2000. Contrafuegos. Barcelona: Anagrama.

Bregman, Rutger. 2017. Utopia for realists: And how we can get there. Londres: Bloomsbury. Brugué, Quim, Xavier Casademont y Eduard Carrera. 2017. La cohesió social a la Garrotxa. Realitats i reptes de futur. Olot: Consorci d'Acció Social de la Garrotxa. Disponible en: https://cutt.ly/jRG5tdk.

Calvo, Cesar y Stefan Dercon. 2009. "Chronic Poverty and All That», en Tonny Addison, David Hulme y Ravi Kanbur (eds.), Poverty Dynamics. Interdisciplined Perspectives. Oxford: Oxford University Press.

Daly, Mary. 2006. Social exclusion as concept and policy template in the European Union. CES Working Paper, 135). Belfast, UK: Center for European Studies, Queen's University.

Ellis, Frank y Philip White. 2000. «Vulnerability to hunger and policy responses in an era of global instability: Southern Africa and the ' 3 Fs Crisis", en Chronic Poverty Research Centre conference on Ten Years Of "War Against Poverty»: What Have We Learned Since.

Eurostat. 2019. Smarter, greener, more inclusive? Indicators to support the Europe 2020 strategy. Luxembourg: Publications Office of the European Union.

Fernández Maíllo, Guillermo. 2019. Informe sobre exclusión y desarrollo social en Cataluña. Resultados de la "Encuesta sobre integración y necesidades sociales 2018». Madrid: Foessa.

Ginsburg, Norman. 1992. Divisions of Welfare. Londres: Sage.

Gorz, Andre 1998. Miserias del presente, riqueza de lo posible. Barcelona: Paidós.

Hickey, Sam y Sarah Bracking. 2005. «Exploring the Politics of Chronic Poverty: From Representation to a Politics of Justice», World Development, 33 (6): 851-865. Disponible en: https://doi.org/10.1016/j.worlddev.2004.09.012.

Hulme, David, Karen Moore y Ander Shepherder. 2001. Chronic Poverty: Meanings and Analytical Framework. CPRC Working Paper, 2. Institute of Development Policy and Management. Disponible en: https://doi.org/10.2139/ssrn.1754546.

Hulme, David y Andrew Shepherd. 2003. "Conceptualizing Chronic Poverty», World Development, 31 (3): 403-423. Disponible en: https://doi.org/10.1016/S0305750X(02)00222-X.

Inglehart, Ronald. 1991. El cambio cultural en las sociedades industriales avanzadas. Madrid: Centro de Investigaciones Sociológicas.

Laparra, Miguel y Begoña Pérez. 2008. «La exclusión social en España: un espacio diverso y disperso en intensa transformación", en VI Informe sobre exclusión y desarrollo social en España. Madrid: Fundación Foessa.

Madanipour, Ali, Mark Shucksmith y Hilary Talbot, 2015. "Concepts of poverty and social exclusion in Europe», Local Economy: The Journal of the Local Economy Policy Unit, 30 (7): 721-741. Disponible en: https://doi.org/10.1177/0269094215601634. 
Malgesini, Graciela. 2014. Informe sobre los sistemas de rentas minimas en España. Disponible en: http://www.ec.europa.eu/social.

Martínez Virto, Lucía., Germán Jaraíz y Miguel Laparra. 2019. «Políticas regionales de inclusión social: de la comparación a la innovación social y el aprendizaje mutuo", Investigaciones Regionales, (44): 5-14.

Moreno, Luis. 2000. Ciudadanos precarios. La última red de protección social. Barcelona: Ariel.

Moruzzi, Mauro y Riccardo Prandini. 2020. Modelli di welfare. Una discussione critica. Franco Angeli Edizioni.

Oosterlynck, Stijn, Andreas Novy, Yuri Kazepov, Pieter Cools, Tatiana Saruis, Bernard Leubolt y Florian Wukovitsch. 2019. «Improving Poverty Reduction: Lessons From the Social Innovation Perspective», en Bea Cantillon, Tim Goedemé y John Hills (eds.), Decent Incomes for All: Improving Policies in Europe. Disponible en: https://doi.org/10.1093/oso/9780190849696.003.0009.

Percy-Smith, Janie (eds.) 2000. Policy Responses to Social Exclusion: Towards inclusion? Buckingham: Open University Press.

Pierson, John. 2002. Tackling Social Exclusion. Londres: Routledge. Disponible en: https://doi.org/10.4324/9780203167427.

Pikkety,Thomas. 2008. L'Économie des Inégaliteés. París: La Découverte.

Pikkety, Thomas. 2014. Capital in the twenty-first century. Cambridge, Massachusetts: The Belknap Press of Harvard University Press.

Piketty, Thomas. 2020. Capital and Ideology. Cambridge: Harvard University Press.

Ranci, Costanzo. 2010. Social Vulnerability in Europe: The New Configuration of Social Risks. Basingstoke: Palgrave Macmillan. Disponible en: https://doi.org/10.1057/ 9780230245778.

Raventós, Daniel y Julie Wark. 2018. Against charity. Chico, CA: AK Press.

Rodríguez, Juan Ramón. 2016. Entreteniendo a los pobres. Albacete: Editorial Bomarzo.

Sen, Amartya. 1984. Resources, Values and Development. Cambridge: Harvard University Press.

Sennett, Richard. 1998. The corrosion of character: The personal consequences of work in the new capitalism. Nueva York: WW Norton and Company.

Standing, Guy. 2011. The Precariat: The New Dangerous Class. Londres: Bloomsbury Academic. Disponible en: https://doi.org/10.5040/9781849664554.

Stiglitz, Joseph. 2013. "The Price of Inequality», New Perspectives Quarterly, 30 (1): 52-53. Disponible en: https://doi.org/10.1111/npqu.11358.

Taylor-Gooby, Peter. 2004. New Risks, New Welfare: The Transformation of the European Welfare State. Oxford: Oxford University Press. Disponible en: https://doi. org/10.1093/019926726X.001.0001.

Tezanos, José Félix. 1999. Tendencias en desigualdad y exclusión social. Madrid: Editorial Sistema.

Townsend, Peter. 2010. "The meaning of poverty», The British Journal of Sociology, (61): 85-102. Disponible en: https://doi.org/10.1111/j.1468-4446.2009.01241.x 


\section{ANEXO 1. TABLA DE PERSONAS ENTREVISTADAS Y CODIFICACIÓN}

\begin{tabular}{lll}
\hline Perfil & Descripción persona entrevistada & Codificación \\
\hline \multirow{2}{*}{ Político } & Presidente consejo comarcal & E1PP \\
\cline { 2 - 3 } & Alcalde & E2PP \\
\cline { 2 - 3 } & Exalcalde & E3PP \\
\cline { 2 - 3 } Pepresentante politico oposición & E4PP \\
\hline \multirow{3}{*}{ Profesionales } & Profesional de la salud & E1P \\
\cline { 2 - 3 } & Profesional de la educación & E2P \\
\cline { 2 - 3 } & Profesional del trabajo social & E3P \\
\cline { 2 - 3 } Socioeconómico & Profesional de la promoción económica y la & E4P \\
\hline & formación ocupacional & E2S \\
\cline { 2 - 3 } & Representante del mundo económico y empresarial del asociacionismo vecinal & E1S \\
\cline { 2 - 3 } & Representate de las entidades de ocio & E3S \\
\cline { 2 - 3 } $\begin{array}{l}\text { Bepresentante de las entidades de solidaridad social } \\
\text { servicios sociales }\end{array}$ & E4S \\
\cline { 2 - 3 } & Beneficiario en una situación cronificada & E1BSS \\
\cline { 2 - 3 } & Beneficiario en una situación de vulnerabilidad & E2BSS \\
\hline
\end{tabular}

Presentado para evaluación: 19 de septiembre de 2020.

Aceptado para publicación: 14 de julio de 2021.

\section{EDUARD CARRERA}

eduard.carrerafossas@udg.edu

Doctorando en Educación por la Universitat de Girona (UdG). Técnico comunitario en el Consorci d'Acció Social de la Garrotxa. Professor asociado del Departamento de Pedagogía de la UdG.

\section{QUIM BRUGUÉ}

q.brugue@udg.edu

Catedrático de Ciencia Política y de la Administración en la UdG. Professor del Departamento de Pedagogía.

\section{XAVIER CASADEMONT}

xavier.casademont@udg.edu

Politólogo. Profesor lector Serra Húnter del Departamento de Pedagogía de la UdG. Coordinador de los estudios de Trabajo Social. 